\title{
The rate of mixing for geodesic and horocycle flows
}

\author{
MARINA RATNER \\ Department of Mathematics, University of California, Berkeley, CA 94720, USA
}

(Received 7 January 1986 and revised 12 May 1986)

Abstract. Using elementary methods we show that for every unitary representation $\pi$ of $G=\operatorname{SL}(2, \mathbb{R})$ with no non-zero invariant vectors the matrix coefficients $\langle\pi(a(t)) v, w\rangle$ of

$$
a(t)=\left(\begin{array}{cc}
e^{t} & 0 \\
0 & e^{-t}
\end{array}\right)
$$

decay exponentially fast for any vectors $v, w$ Hölder continuous in the direction of the rotation subgroup of $G$.

Let $G$ denote the group $\operatorname{SL}(2, \mathbb{R})$ and let $\Gamma$ be a lattice in $G$. This means that $\Gamma$ is a discrete subgroup of $G$ and the measure $\mu$ on the quotient space $M=\Gamma \backslash G$ derived from the Haar measure on $G$ is finite. We assume that $\mu$ is normalized, i.e. $\mu(M)=1$.

The geodesic and the horocycle flows on $M$ are defined by

$$
\begin{aligned}
& g_{t}(\Gamma g)=\Gamma g\left(\begin{array}{cc}
e^{t} & 0 \\
0 & e^{-t}
\end{array}\right) \\
& h_{t}(\Gamma g)=\Gamma g\left(\begin{array}{ll}
1 & 0 \\
t & 1
\end{array}\right)
\end{aligned}
$$

$g \in G, t \in \mathbb{R}$.

These flows can be viewed geometrically as the geodesic and the horocycle flows on the unit tangent bundle of a surface of constant negative curvature with finite volume.

The flows preserve $\mu$ and are mixing in $(M, \mu)$. This means that if $f, \phi \in L_{2}(M, \mu)$ and $\langle f, 1\rangle=0$ then $B_{\phi, f}(t)=\left\langle\phi, f \circ g_{t}\right\rangle$ and $C_{\phi, f}(t)=\left\langle\phi, f \circ h_{t}\right\rangle$ decay to zero when $t \rightarrow \infty$.

We shall investigate these rates of decay.

In the Fall of 1983 I asked Calvin Moore if he could prove that if $\phi, f \in L_{2}(M, \mu)$ are Hölder continuous on $M$ and $\langle f, 1\rangle=0$ then

$$
\left|B_{\phi, f}(t)\right| \leq C e^{-\alpha|t|}
$$

for all $t \in \mathbb{R}$ and some $C, \alpha>0$, depending only on $\phi, f$. Moore said that he found a proof of this for any $\phi, f \in L_{2}(M, \mu)$ Hölder continuous in the direction of the rotation group $K$ (see definition below) with the Hölder exponents greater than $\frac{1}{2}$. For certain classes of analytic functions this exponential decay has been found by 
a number of authors (see [1, p. 136] and [2], [14], [16]). Moore's arguments, (the details of Moore's argument are not yet available since his manuscript is still in preparation), as well as those in [1], [14], [16], which actually apply to more general semisimple groups $G$, rely crucially on a substantial amount of group representation theory.

In this paper we give a different and rather elementary proof of this exponential decay with no restrictions on the Hölder exponents of $\phi, f$. Although we also turn to group representation theory we use merely the basic theorem about decomposition of an arbitrary representation into irreducible ones. It seems likely that this approach may generalize to geodesic flows on unit tangent bundles of compact surfaces of variable negative curvature. In this connection the paper [5] of Guillemin and Kazhdan is suggestive.

We begin with some basic concepts and definitions (see [4], [11]).

Let $\mathbf{H}$ be a complex separable Hilbert space, $U(\mathbf{H})$ the group of all unitary transformations of $\mathbf{H}$ onto itself and $T: G \rightarrow U(\mathbf{H}), T(g)=T_{g} \in U(\mathbf{H})$ a unitary representation of $G$ in $\mathbf{H}$. We shall denote the Hilbert space $\mathbf{H}$ by $\mathbf{H}(T)$.

An element $v \in \mathbf{H}(T)$ is called a $C^{k}$-vector for $T, k=0,1, \ldots, \infty$ if $g \rightarrow T_{g}(v)$ is a $C^{k}$-map from $G$ to the Hilbert space $\mathbf{H}(T)$ (see [10]). The space of $C^{\infty}$-vectors is dense in $\mathbf{H}(T)$.

The Lie algebra $g$ of $G$ consists of all $2 \times 2$ real matrices with trace 0 . Let $X \in g$ and $v$ be a $C^{1}$-vector in $\mathbf{H}(T)$. The Lie derivative $L_{X} v$ is defined by

$$
L_{X} v=\lim _{t \rightarrow 0} \frac{T(\exp t X) v-v}{t} .
$$

It satisfies

$$
\left\langle L_{X} v, w\right\rangle=-\left\langle v, L_{X} w\right\rangle
$$

for all $C^{1}$-vectors $v, w \in \mathbf{H}(T)$ and all $X \in g$.

We choose the following basis in $g$.

$$
W=\left(\begin{array}{rr}
0 & 1 \\
-1 & 0
\end{array}\right), \quad Q=\left(\begin{array}{rr}
1 & 0 \\
0 & -1
\end{array}\right), \quad V=\left(\begin{array}{ll}
0 & 1 \\
1 & 0
\end{array}\right)
$$

We have

$$
\exp t W=\left(\begin{array}{cc}
\cos t & \sin t \\
-\sin t & \cos t
\end{array}\right), \quad \exp t Q=\left(\begin{array}{cc}
e^{t} & 0 \\
0 & e^{-t}
\end{array}\right), \quad \exp t V=\left(\begin{array}{cc}
c h t & s h t \\
s h t & c h t
\end{array}\right)
$$

and

$$
[Q, W]=2 V, \quad[Q, V]=2 W, \quad[W, V]=2 Q .
$$

The Casimir operator $\Omega_{T}$ is defined on $C^{2}$-vectors in $\mathbf{H}(T)$ by the formula

$$
\Omega_{T}=\left(L_{V}^{2}+L_{Q}^{2}-L_{W}^{2}\right) / 4 \text {. }
$$

It satisfies

$$
\left\langle\Omega_{T} v, w\right\rangle=\left\langle v, \Omega_{T} w\right\rangle
$$

for all $C^{2}$-vectors $v, w \in \mathbf{H}(T)$.

It is a fact that the closure of $\Omega_{T}$ is self-adjoint. $\Omega_{T}$ commutes with every $L_{X}$, $X \in g$ on $C^{3}$-vectors in $\mathbf{H}(T)$. It also commutes with all $T_{g}, g \in G$ and if $T$ is 
irreducible then $\Omega_{T}$ is a scalar multiple of the identity, i.e.

$$
\Omega_{T} v=\lambda v
$$

for some $\lambda=\lambda(T) \in \mathbb{R}$ and all $C^{2}$-vectors $v \in \mathbf{H}(T)$.

The spectrum $\Lambda\left(\Omega_{T}\right)$ plays a crucial role in our analysis.

Let now $\Gamma$ be a discrete subgroup of $G, M=\Gamma \backslash G$ and $\mathbf{H}=L_{2}(M, \mu)$. For $g \in G$ let $T_{\mathrm{g}}$ be the unitary transformation of $\mathbf{H}$ onto itself defined by

$$
\left(T_{g} f\right)(\Gamma z)=f(\Gamma z \cdot g), \quad z \in G, f \in \mathbf{H} .
$$

The homomorphism $T: g \rightarrow T_{g}$ is called the regular representation of $G$ on $L_{2}(M, \mu)$.

Let $K$ be the rotation subgroup of $G$

$$
K=\left\{r(\theta)=\left(\begin{array}{rr}
\cos \theta & \sin \theta \\
-\sin \theta & \cos \theta
\end{array}\right): \theta \in \mathbb{R}\right\}
$$

and let $H$ denote the upper half plane $\operatorname{Im} z>0, z=x+i y$, equipped with the hyperbolic metric $d s^{2}=\left(d x^{2}+d y^{2}\right) / y^{2}$. The curvature of $H$ equals -1 at every $z \in H$.

The quotient space $G / K$ is diffeomorphic to $H$ via the map $\phi: G / K \rightarrow H$ defined by

$$
\phi(g K)=g(i)
$$

where

$$
g(i)=\frac{a i+b}{c i+d} \quad \text { and } \quad g=\left(\begin{array}{ll}
a & b \\
c & d
\end{array}\right) \in G .
$$

The diffeomorphism $\phi^{-1}$ carries to $G / K$ the coordinate system and the hyperbolic Riemannian metric from $H$. In this metric the quotient space $S=\Gamma \backslash G / K$ is a surface of constant negative curvature.

Let $\mathbf{H}^{\prime}=\left\{f \in L_{2}(M, \mu): f\right.$ is constant on orbits of $\left.K\right\}$. Each function in $\mathbf{H}^{\prime}$ is in fact a function on $S$ and $\mathbf{H}^{\prime}$ can be identified with $L_{2}(S)$. Let $\Omega$ be the Casimir operator associated with the regular representation of $G$ on $L_{2}(M, \mu)$. One can show [11] that $\Omega$ restricted to $C^{2}$-vectors of $\mathbf{H}^{\prime}$ coincides with the Laplace operator $\Delta$ on $L_{2}(S)$ which in coordinates $(x, y)$ has the form

$$
\Delta=y^{2}\left(\frac{\partial^{2}}{\partial x^{2}}+\frac{\partial^{2}}{\partial y^{2}}\right) \text {. }
$$

Let $T$ be a unitary representation of $G$ in $\mathbf{H}(T)$.

Definition. A vector $v \in \mathbf{H}(T)$ is called a $K^{p}$-vector, $p>0$, if $\theta \rightarrow T_{r(\theta)} v$ is a $C^{p}$-map from $\mathbb{R}$ to $\mathbf{H}(T)$. We shall denote by $K(T, p), p>0$ the space of all $K^{p}$-vectors in $\mathbf{H}(T)$.

For $0<p<1$ the expression $v \in K(T, p)$ means that $v$ is Hölder continuous in the direction of $K$ with the Hölder exponent $p$, i.e.

$$
\sup _{\theta \neq 0} \frac{\left\|T_{r(\theta)} v-v\right\|}{|\theta|^{p}}<\infty .
$$

For $n \leq p<n+1, n \in \mathbb{Z}^{+}$the expression $v \in K(T, p)$ means that the $n$th derivative 
$L_{W}^{n} v$ exists and it belongs to $K(T, p-n)$ if $p \neq n$. Write

$$
\begin{gathered}
a(t)=\left(\begin{array}{cc}
e^{t} & 0 \\
0 & e^{-t}
\end{array}\right), \quad u(t)=\left(\begin{array}{ll}
1 & 0 \\
t & 1
\end{array}\right) \\
v \circ g=T_{g} v, \quad v \in \mathbf{H}(T), g \in G .
\end{gathered}
$$

For $\lambda \in\left(-\frac{1}{4}, 0\right)$ let

$$
r(\lambda)=-1+\sqrt{1+4 \lambda} \in(-1,0) .
$$

$r(\lambda)$ satisfies the quadratic equation

$$
k^{2}+2 k-4 \lambda=0 .
$$

THEOREM 1. Let $T$ be a non-trivial irreducible unitary representation of $G$ in $\mathbf{H}(T)$ and let $\lambda=\lambda(T)$. Let $v, w \in K(T, p), \quad p>0$ and $B(t)=\langle v, w \circ a(t)\rangle, \quad C(t)=$ $\langle v, w \circ u(t)\rangle$. Then for all $|t| \geq 1$

$$
|B(t)| \leq C\left(b_{\lambda}(|t|)\right)^{\alpha(p)}, \quad|C(t)| \leq D\left(b_{\lambda}(\ln |t|)\right)^{\alpha(p)},
$$

where

(1) $b_{\lambda}(t)=\min \left\{t e^{-t}, e^{-t}\left(1+|1+4 \lambda|^{-1 / 2}\right)\right\} \quad$ if $\lambda \leq-\frac{1}{4}$;

(2) $b_{\lambda}(t)=\min \left\{t e^{r(\lambda) t}, e^{r(\lambda) t}|1+4 \lambda|^{-1 / 2}\right\} \quad$ if $\lambda \in\left(-\frac{1}{4}, 0\right)$;

(3) $b_{\lambda}(t)=t e^{-2 t} \quad$ if $\lambda \geq 0$.

Here

$$
\alpha(p)=\left\{\begin{array}{cl}
1 & p \geq 3 \\
\frac{2 p}{2 p+1} & 2 \leq p<3 \\
\frac{2 p}{2 p+3} & 1 \leq p<2 \\
\frac{p}{p+3} & 0<p<1
\end{array}\right.
$$

and $C, D>0$ depend only on $v, w$ and $p$ (not on $\lambda$ ).

The proof of theorem 1 is elementary. We choose an appropriate orthonormal basis $\left\{\phi_{n}\right\}$ in $\mathbf{H}(T)$, write a differential equation for $B_{n, m}(t)=\left\langle\phi_{n}, \phi_{m} \circ a(t)\right\rangle$ and estimate its solution. Then we use harmonic analysis. In fact, our proof gives not only the decay rate but also several additional terms in the asymptotic expansion of $B(t)$ and $C(t)$ when $t \rightarrow \infty$.

According to Bargmann's classification of irreducible unitary representations of $G$ the eigenvalue $\lambda=\lambda(T)$ of $\Omega_{T}$ has the form

$$
\lambda(T)=\frac{s^{2}-1}{4},
$$

where $s$ is either pure imaginary (the principal series) or $s \in(-1,1), s \neq 0$ (the complementary series) or $s$ is an integer (the discrete series). If $\lambda \in\left(-\frac{1}{4}, 0\right)$ then $0 \neq s \in(-1,1)$ and

$$
r(\lambda)=-1+|s|
$$

We do not use Bargmann's classification in this paper. 
Let now $\Gamma$ be a discrete subgroup of $G, M=\Gamma \backslash G, S=\Gamma \backslash G / K$ and $\Delta$ be the Laplace operator on $L_{2}(S)$. Theorem 1 shows that the number $-\frac{1}{4}$ plays a special role in our analysis of the decay rate just as it does in the spectral decomposition of $\Delta$.

It is well known [11] that a number $\lambda \in\left(-\frac{1}{4}, 0\right)$ belongs to the spectrum $\Lambda=\Lambda(\Omega)$ of $\Omega$ on $L_{2}(M, \mu)$ if and only if it belongs to the spectrum $\Lambda(\Delta)$ of $\Delta$ on $L_{2}(S)$. Write

$$
\begin{aligned}
& A(\Gamma)=\Lambda(\Delta) \cap\left(-\frac{1}{4}, 0\right) \\
& \beta(\Gamma)=\sup A(\Gamma), \quad \text { if } A(\Gamma) \neq \varnothing \\
& \sigma(\Gamma)=r(\beta(\Gamma))=-1+\sqrt{1+4 \beta(\Gamma)} .
\end{aligned}
$$

It is a fact (see [3], [9], [11]) that if $\Gamma$ is a lattice in $G$ then $A(\Gamma)$ is finite. In this case $\beta(\Gamma)<0$ if $A(\Gamma) \neq \varnothing$.

We prove the following:

THEOREM 2. Let $\Gamma$ be a lattice in $G, M=\Gamma \backslash G$ and $T$ be the regular representation of $G$ on $L_{2}(M, \mu)$. Let $v, w \in K(T, p), p>0,\langle w, 1\rangle=0$ and $B(t)=\langle v, w \circ a(t)\rangle$, $C(t)=\langle v, w \circ u(t)\rangle$. Then there exists $t_{0}=t_{0}(\Gamma)>0$ s.t. for all $|t| \geq t_{0}$ and some $C, D>0$

where

$$
|B(t)| \leq C(b(|t|))^{\alpha(p)}, \quad|C(t)| \leq D(b(\ln |t|))^{\alpha(p)},
$$

(1) $b(t)=e^{\sigma(\Gamma) t} \quad$ if $A(\Gamma) \neq \varnothing$

(2) $b(t)=e^{-t}$ if $A(\Gamma)=\varnothing$, sup $\left(\Lambda \cap\left(-\infty,-\frac{1}{4}\right)\right)<-\frac{1}{4}$ and $-\frac{1}{4}$ is not an eigenvalue of $\Omega$;

(3) $b(t)=t e^{-t}$ if $A(\Gamma)=\varnothing$ and either $\sup \left(\Lambda \cap\left(-\infty,-\frac{1}{4}\right)\right)=-\frac{1}{4}$ or $-\frac{1}{4}$ is an eigenvalue of $\Omega$;

and $\alpha(p)$ is as in theorem 1 .

Let us note that if $M$ is compact then $\Lambda(\Omega)$ is discrete and therefore

$$
\sup \left(\Lambda \cap\left(-\infty,-\frac{1}{4}\right)\right)<-\frac{1}{4} \text {. }
$$

Corollary 1. If $v, w$ are any two functions in $L_{2}(S)$ with $\langle w, 1\rangle=0$ then theorem 2 holds for $v, w$ with $\alpha(p)=1$. In fact, $\Lambda=\Lambda(\Omega)$ in (2) and (3) can be replaced by $\Lambda(\Delta)$.

Selberg proved that if $\Gamma=\operatorname{SL}(2, \mathbb{Z})$ then $A(\Gamma)=\varnothing$. We get the following:

Corollary 2. Let $\Gamma=\operatorname{SL}(2, \mathbb{Z})$. Then $b(t)=t e^{-t}$ in theorem 2 .

When $M=\Gamma \backslash G$ is compact, $L_{2}(M, \mu)$ is a direct sum of irreducible subspaces of $T$. To prove theorem 2 we simply apply harmonic analysis and theorem 1 . This makes it plausible that our proof may generalize in various ways.

The non-compact case is not much different from the compact case. We have only to decompose representations as direct integrals instead of direct sums. In fact, theorem 2 follows from a much more general theorem 3 .

THEOREM 3. Let $T$ be a unitary representation of $G$ having no non-zero invariant vectors in $\mathbf{H}(T)$. Write

$$
\begin{aligned}
& \Lambda=\Lambda\left(\Omega_{T}\right), \quad A(T)=\Lambda \cap\left(-\frac{1}{4}, 0\right) \\
& \beta(T)=\sup A(T) \quad \text { if } A(T) \neq \varnothing \\
& \sigma(T)=-1+\sqrt{1+4 \beta(T)}
\end{aligned}
$$


Assume that $\beta(T)<0$ if $A(T) \neq \varnothing$. Let $v, w \in K(T, p), p>0, B(t)=\langle v, w \circ a(t)\rangle$, $C(t)=\langle v, w \circ u(t)\rangle$. Then there exists $t_{0}=t_{0}(T)>0$ s.t. for all $|t| \geq t_{0}$ and some $C, D>0$

$$
|B(t)| \leq C(b(|t|))^{\alpha(p)}, \quad|C(t)| \leq D(b(\ln |t|))^{\alpha(p)}
$$

where

(1) $b(t)=e^{\sigma(T) t} \quad$ if $A(T) \neq \varnothing$

(2) $b(t)=e^{-t}$ if $A(T)=\varnothing$, sup $\left(\Lambda \cap\left(-\infty,-\frac{1}{4}\right)\right)<-\frac{1}{4}$ and $-\frac{1}{4}$ is not an eigenvalue of $\Omega_{T}$;

(3) $b(t)=t e^{-t}$ if $A(T)=\varnothing$ and either $\sup \left(\Lambda \cap\left(-\infty,-\frac{1}{4}\right)\right)=-\frac{1}{4}$ or $-\frac{1}{4}$ is an eigenvalue of $\Omega_{T}$;

(4) $b(t)=t e^{-2 t}$ if $\Lambda \subset[0, \infty)$;

and $\alpha(p)$ is as in theorem 1 .

It was shown in [12] and [15] that if $\Gamma$ is a finitely generated discrete subgroup of $G$ then $A(\Gamma)$ is finite. We get the following generalization of theorem 2 .

CoRollary 3. Let $\Gamma$ be a finitely generated discrete subgroup of $G$ with $\mu(M)=\infty$, $M=\Gamma \backslash G$ and let $T$ be the regular representation of $G$ in $L_{2}(M, \mu)$. Then the conclusion of theorem 2 holds for all $v, w \in K(T, p), p>0$.

I wish to thank Paul Chernoff, Yitzhak Katznelson and Steve Zelditch for useful conversations on various aspects of the problem.

This research was partially supported by NSF Grant DMS-84-20770 and Miller Institute for Basic Research, Berkeley.

\section{Harmonic Analysis}

We begin by recalling some basic facts about direct integrals of representations (see [13]). Let $(Y, \nu)$ be a standard measure space and for each $y \in Y$, let $\pi_{y}$ be a non-trivial irreducible unitary representation of $G$ in a complex separable Hilbert space $\mathbf{H}(y)$. Let

$$
\Psi(y)=\left\{\psi_{n}(y), n \in \mathbb{Z}\right\}
$$

be an orthonormal basis in $\mathbf{H}(y)$ s.t. the inner product $\left\langle\pi_{y}(g) \psi_{n}(y), \psi_{m}(y)\right\rangle$ is a measurable function of $(g, y), g \in G, y \in Y$ for all $n, m \in \mathbb{Z}$.

We say that a function $f$ on $Y$ with $f(y) \in \mathbf{H}(y)$ is $\Psi$-measurable if $\left\langle f(y), \psi_{n}(y)\right\rangle$ is a measurable function of $y$ for all $n \in \mathbb{Z}$.

Let $\mathbf{H}$ be the set of all $\Psi$-measurable functions $f$ with

$$
\int_{Y}\|f(y)\|^{2} d \nu(y)<\infty .
$$

As usual after identifying functions that differ on a set of $\nu$-measure zero $\mathbf{H}$ becomes a separable Hilbert space under the inner product

The direct integral

$$
\langle f, h\rangle=\int_{Y}\langle f(y), h(y)\rangle d \nu(y) .
$$

$$
\pi=\int_{Y} \pi_{y} d \nu(y)
$$


is defined as the unitary representation of $G$ on $\mathbf{H}$ given by

$$
(\pi(g) f)(y)=\left(\pi_{y}(g)\right)(f(y))
$$

$g \in G, y \in Y, f \in \mathbf{H}$.

Fact 1.1 (see [13]). Let $T$ be a unitary representation of $G$ having no non-zero invariant vectors in $\mathbf{H}(T)$. Then $T$ is unitary equivalent to a direct integral $\pi$ just described.

Suppose that a vector $v \in \mathbf{H}(T)$ is an eigenvector for the action of $K$ on $\mathbf{H}(T)$. Then there is an integer $n$ s.t.

$$
T_{r(\theta)} v=e^{i n \theta} v
$$

for all $\theta \in \mathbb{R}$.

For $n \in \mathbb{Z}$ denote

$$
\mathbf{H}_{n}(T)=\left\{v \in \mathbf{H}(T): T_{r(\theta)} v=e^{i n \theta} v, \theta \in \mathbb{R}\right\} .
$$

It is a fact (see [11]) that $\mathbf{H}(T)=\sum \mathbf{H}_{n}(T)$ is the direct orthogonal sum of $\mathbf{H}_{n}(T)$. If $T$ is irreducible then $\operatorname{dim} \mathbf{H}_{n}(T)=0$ or 1 .

Let now $\pi$ be the direct integral of representations $\pi_{y}, y \in Y$ defined above. We shall show that the basis $\Psi(y), y \in Y$ in the definition of $\pi$ can be replaced by a basis consisting of eigenvectors of $K$ in $\mathbf{H}(y)$.

LeMMA 1.1. There exists a set $\Phi_{0}(y)=\left\{\phi_{n}(y), n \in \mathbb{Z}\right\}, y \in Y$ with $0 \neq \phi_{n}(y) \in \mathbf{H}_{n}(y)$ if $\operatorname{dim} \mathbf{H}_{n}(y)=1, \phi_{n}(y)=0$ if $\operatorname{dim} \mathbf{H}_{n}(y)=0$ such that the set $\Phi(y)=\Phi_{0}(y)-\{0\}$ forms an orthonormal basis in $\mathbf{H}(y)$ and a function $f$ on $Y$ is $\Psi$-measurable if and only if it is $\Phi$-measurable.

Proof. We have $\mathbf{H}(\pi)=\sum \mathbf{H}_{n}(\pi)$. For $n \in \mathbb{Z}$ let $\left\{e_{k}^{n}: k=1,2, \ldots\right\}$ be an orthonormal basis in $\mathbf{H}_{n}(\pi)$ and let

$$
\begin{aligned}
& Y_{k}^{n}=\left\{y \in Y: e_{k}^{n}(y) \neq 0\right\} \\
& \bar{Y}_{k}^{n}=Y_{k}^{n}-\bigcup_{i=1}^{k-1} Y_{i}^{n} \\
& Y_{n}=\bigcup_{k=1}^{\infty} \bar{Y}_{k}^{n} .
\end{aligned}
$$

All these sets are measurable subsets of $Y$ and $Y_{n}$ is a disjoint union. It is clear that

$$
\nu\left(Y-\bigcup_{n=1}^{\infty} Y_{n}\right)=0 .
$$

This says that we can assume without loss of generality that $Y=\bigcup_{n=1}^{\infty} Y_{n}$.

For $n \in \mathbb{Z}$ let

$$
\phi_{n}(y)= \begin{cases}\frac{e_{k}^{n}(y)}{\left\|e_{k}^{n}(y)\right\|} & \text { if } y \in \bar{Y}_{k}^{n} \\ 0 & \text { if } y \in Y-Y_{n}\end{cases}
$$

$\phi_{n}(y) \in \mathbf{H}_{n}(y), n \in \mathbb{Z}, y \in Y$. It is clear that $\left\langle\phi_{n}(y), \psi_{m}(y)\right\rangle$ is a measurable function of $y$ for all $n, m \in \mathbb{Z}$. Let

$$
\Phi_{0}(y)=\left\{\phi_{n}(y): n \in \mathbb{Z}\right\}
$$


We claim that $\Phi(y)$ forms an orthonormal basis in $\mathbf{H}(y)$. Indeed, let $\overline{\mathbf{H}}(y)$ be the closed subspace of $\mathbf{H}(y)$, spanned by $\Phi(y)$. It is clear that

$$
\overline{\mathbf{H}}(y)=\{f(y): f \in \mathbf{H}(\pi)\}
$$

and

$$
\left(\pi_{y}(g)\right)\left(\phi_{n}(y)\right) \in \overline{\mathbf{H}}(y)
$$

for all $y \in Y, g \in G, n \in \mathbb{Z}$. This implies that $\overline{\mathbf{H}}(y)$ is $\pi_{y}$-invariant and therefore $\overline{\mathbf{H}}(y)=\mathbf{H}(y)$, since $\pi_{y}$ is irreducible, $y \in Y$.

It is clear from the construction of $\Phi(y)$ that a function $f$ is $\Phi$-measurable if and only if it is $\Psi$-measurable.

Henceforth we shall always assume that $\pi$ is the direct integral of representations $\pi_{y}$ on $\mathbf{H}(y), y \in Y$ defined above with a basis $\Phi(y)=\left\{\phi_{n}(y): n \in \mathbb{Z}\right\}-\{0\}$ in $\mathbf{H}(y)$ such that

$$
\begin{aligned}
0 \neq \phi_{n}(y) \in \mathbf{H}_{n}(y) & \text { if } \operatorname{dim} \mathbf{H}_{n}(y)=1, \\
\phi_{n}(y)=0 & \text { if } \operatorname{dim} \mathbf{H}_{n}(y)=0,
\end{aligned}
$$

$n \in \mathbb{Z}, y \in Y$. Thus

$$
\pi_{y}(r(\theta)) \phi_{n}(y)=e^{i n \theta} \phi_{n}(y)
$$

for all $n \in \mathbb{Z}, y \in Y, \theta \in \mathbb{R}$.

We are interested in $K^{p}$-vectors in $\mathbf{H}(\pi)$. If $f \in K(\pi, 1)$, then the derivative of $f$ in the direction of $K$ exists and is $L_{W} f \in \mathbf{H}(\pi)$. We shall denote by $L_{w}^{y}, y \in Y$ the derivatives in $\mathbf{H}(y)$.

LEMMA 1.2. Let $f \in K(\pi, 1)$. Then for almost every (a.e.) $y \in Y$

(1) $\left\langle\left(L_{W} f\right)(y), \phi_{n}(y)\right\rangle=\operatorname{in}\left\langle f(y), \phi_{n}(y)\right\rangle$;

(2) $f(y) \in K\left(\pi_{y}, 1\right)$;

(3) $\left(L_{w} f\right)(y)=L_{w}^{y}(f(y))$.

Proof. Let $Y^{\prime}$ be a measurable subset of $Y$ with $\nu\left(Y^{\prime}\right)>0$ and let $\phi_{n}^{\prime}, n \in \mathbb{Z}$ be defined on $Y$ by

$$
\phi_{n}^{\prime}(y)= \begin{cases}\phi_{n}(y) & y \in Y^{\prime} \\ 0 & y \notin Y^{\prime}\end{cases}
$$

Then $\phi_{n}^{\prime} \in \mathbf{H}(\pi)$ and

$$
\begin{aligned}
\left\|\frac{\pi(r(\theta)) \phi_{n}^{\prime}-\phi_{n}^{\prime}}{\theta}-i n \phi_{n}^{\prime}\right\|^{2} & =\int_{Y^{\prime}}\left\|\frac{\pi_{y}(r(\theta)) \phi_{n}(y)-\phi_{n}(y)}{\theta}-i n \phi_{n}(y)\right\|^{2} d \nu(y) \\
& =\int_{Y^{\prime}}\left|\frac{e^{i n \theta}-1}{\theta}-i n\right|^{2}\left\|\phi_{n}(y)\right\|^{2} d \nu(y) \\
& =\left|\frac{e^{i n \theta}-1}{\theta}-i n\right|^{2} \nu\left\{y \in Y^{\prime}: \phi_{n}(y) \neq 0\right\} .
\end{aligned}
$$

This implies that $\phi_{n}^{\prime} \in K(\pi, 1)$, and

$$
L_{W} \phi_{n}^{\prime}=\operatorname{in} \phi_{n}^{\prime}, \quad n \in \mathbb{Z} \text {. }
$$


We have

$$
\left\langle L_{W} f, \phi_{n}^{\prime}\right\rangle=-\left\langle f, L_{W} \phi_{n}^{\prime}\right\rangle=\operatorname{in}\left\langle f, \phi_{n}^{\prime}\right\rangle
$$

or

$$
\int_{Y^{\prime}}\left\langle\left(L_{W} f\right)(y), \phi_{n}(y)\right\rangle d \nu(y)=\text { in } \int_{Y^{\prime}}\left\langle f(y), \phi_{n}(y)\right\rangle d \nu(y) .
$$

This is true for all $Y^{\prime} \subset Y$ with positive $\nu$-measure. Therefore

$$
\left\langle\left(L_{W} f\right)(y), \phi_{n}(y)\right\rangle=i n\left\langle f(y), \phi_{n}(y)\right\rangle
$$

for a.e. $y \in Y$ and all $n \in \mathbb{Z}$. Also

$$
\int_{Y}\left(\sum_{n \in \mathbf{Z}} n^{2}\left|\left\langle f(y), \phi_{n}(y)\right\rangle\right|^{2}\right) d \nu(y)<\infty
$$

since $L_{W} f \in \mathbf{H}(\pi)$. This implies that

$$
\sum_{n \in \mathbf{Z}} n^{2}\left|\left\langle f(y), \phi_{n}(y)\right\rangle\right|^{2}<\infty
$$

for a.e. $y \in Y$. For these $y$ the vector $f(y) \in K\left(\pi_{y}, 1\right)$ and

$$
\left(L_{W} f\right)(y)=L_{W}^{y}(f(y)) .
$$

Corollary 1.1. Let $f \in K(\pi, m)$ for some integer $m>0$. Then

$$
\left\|L_{W}^{k} f\right\|^{2}=\int_{Y}\left(\sum_{n \in \mathbf{Z}} n^{2 k}\left|\left\langle f(y), \phi_{n}(y)\right\rangle\right|^{2}\right) d \nu(y)<\infty .
$$

for all $k=1, \ldots, m$.

Now let $f \in K(\pi, p)$ with $0<p<1$. Then

$$
\|\pi(r(\theta)) f-f\| \leq C|\theta|^{p}
$$

for some $C>0$ and all $\theta \in \mathbb{R}$. Write

$$
\left\langle f(y), \phi_{n}(y)\right\rangle=c_{n, f}(y) .
$$

The proof of the following lemma is completely analogous to the proof of Bernstein's theorem in $[8]$.

LeMmA 1.3. Let $f \in K(\pi, p)$ with $0<p<1$. Then

$$
Q(t)=\int_{Y}\left(\sum_{|n| \geq t}\left|c_{n, f}(y)\right|^{2}\right) d \nu(y) \leq D t^{-2 p}
$$

for some $D>0$ and all $t \geq 1$.

Proof. For $m \in \mathbb{Z}^{+} \cup\{0\}$ let

$$
\theta_{m}=2 \pi / 3 \cdot 2^{m}
$$

Then for all $n$ with $2^{m} \leq|n| \leq 2^{m+1}$ we have

$$
\left|e^{i n \theta_{m}}-1\right| \geq \sqrt{3} \text {. }
$$

Also

$$
\left\|\pi\left(r\left(\theta_{m}\right)\right) f-f\right\|^{2}=\int_{Y}\left(\sum_{n \in \mathbf{Z}}\left|e^{i n \theta_{m}}-1\right|^{2}\left|c_{n, f}(y)\right|^{2}\right) d \nu(y) \leq C^{2}\left|\theta_{m}\right|^{2 p}
$$

by (1.2). 
We have using (1.3) and (1.4)

$$
\begin{aligned}
E_{m} & =\int_{Y}\left(\sum_{2^{m} \leq|n| \leq 2^{m+1}}\left|c_{n, f}(y)\right|^{2}\right) d \nu(y) \\
& \leq \int_{Y}\left(\sum_{2^{m} \leq|n| \leq 2^{m+1}}\left|e^{i n \theta_{m}}-1\right|^{2}\left|c_{n, f}(y)\right|^{2}\right) d \nu(y) \\
& \leq\left\|\pi\left(r\left(\theta_{m}\right)\right) f-f\right\|^{2} \leq C^{2} \theta_{m}^{2 p}=C^{2}\left(\frac{2 \pi}{3 \cdot 2^{m}}\right)^{2 p}
\end{aligned}
$$

This is true for all $m \in \mathbb{Z}^{+} \cup\{0\}$.

For a given $t \geq 1$ let $m_{0}$ be s.t.

Then

$$
2^{m_{0}} \leq t<2^{m_{0}+1}
$$

$$
Q(t) \leq \sum_{m=m_{0}}^{\infty} E_{m} \leq D 2^{-2 p\left(m_{0}+1\right)} \leq D t^{-2 p}
$$

for some $D>0$ by (1.5). This completes the proof.

Corollary 1.2. Let $p>0$ and $f \in K(\pi, p)$. Then

$$
Q(t)=\int_{Y}\left(\sum_{|n| \geq t}\left|c_{n, f}(y)\right|^{2}\right) d \nu(y) \leq \bar{D} t^{-2 p}
$$

for all $t \geq 1$ and some $\bar{D}>0$, depending only on $f$.

Proof. For $0<p<1$ this is just lemma 1.3. Let $p \geq 1, k=k(p)=[p]$ and

$$
c_{n, f}^{k}(y)=\left\langle L_{W}^{k} f(y), \phi_{n}(y)\right\rangle \text {. }
$$

We have using lemmas 1.2 and 1.3

$$
\begin{aligned}
Q(t)=\int_{Y}\left(\sum_{|n| \geq t} \frac{\left|c_{n, f}^{k}(y)\right|^{2}}{n^{2 k}}\right) d \nu(y) & \leq t^{-2 k} \int_{Y}\left(\sum\left|c_{n, f}^{k}(y)\right|^{2}\right) d \nu(y) \\
& \leq \bar{D} t^{-2 k} \cdot t^{-2(p-k)}=\bar{D} t^{-2 p}
\end{aligned}
$$

\section{Proofs of theorems 1 and 3}

Let $T$ be a non-trivial irreducible unitary representation of $G$ in $\mathbf{H}(T)$ and let $\Omega=\Omega_{T}$ be the Casimir operator associated with $T$. We have

$$
\Omega v=\lambda v
$$

for some $\lambda=\lambda(T) \in \mathbb{R}$ and all $C^{2}$-vectors $v \in \mathbf{H}(T)$.

As we mentioned above $\mathbf{H}(T)$ has an orthonormal basis $\left\{\phi_{n}, n \in \mathbb{Z}\right\}-\{0\}$ with $0 \neq \phi_{n} \in \mathbf{H}_{n}(T)$ if $\operatorname{dim} \mathbf{H}_{n}(T)=1$ and $\phi_{n}=0$ if $\operatorname{dim} \mathbf{H}_{n}(T)=0$. We have

$$
T(r(\theta)) \phi_{n}=e^{i n \theta} \phi_{n}
$$

for all $n \in \mathbb{Z}, \theta \in \mathbb{R}$.

It is a fact that $\phi_{n}$ are $C^{\infty}$-vectors in $\mathbf{H}(T)$ (see [11]). Expression (2.1) shows that

$$
L_{W} \phi_{n}=\operatorname{in} \phi_{n} \quad n \in \mathbb{Z} \text {. }
$$

(see (1) for definition of $W$ and $V, Q$ below). Also

$$
\Omega \phi_{n}=\lambda \phi_{n}
$$

for all $n \in \mathbb{Z}$. 
Denote

$$
B_{n, m}(t)=\left\langle\phi_{n}, \phi_{m} \circ a(t)\right\rangle
$$

$n, m \in \mathbb{Z}$. Here

$$
\phi_{m} \circ a(t)=T_{a(t)} \phi_{m}
$$

by (2).

The function $B_{n, m}(t)$ is $C^{\infty}$ in $t$ and

$$
\begin{aligned}
& B_{n, m}^{\prime}(t)=\left\langle\phi_{n},\left(L_{Q} \phi_{m}\right) \circ a(t)\right\rangle \\
& B_{n, m}^{\prime \prime}(t)=\left\langle\phi_{n},\left(L_{Q}^{2} \phi_{m}\right) \circ a(t)\right\rangle .
\end{aligned}
$$

LEMMA 2.1. The function $B_{n, m}(t)$ satisfies the following differential equation for $t \geq 1$

where

$$
y^{\prime \prime}+2 y^{\prime}-4 \lambda y=f_{1}(t)+f_{2}(t)
$$

$$
\begin{aligned}
& f_{1}(t)=\left(2 e^{2 t} \operatorname{sh} 2 t\right)^{-1} y^{\prime}(t) \\
& f_{2}(t)=y(t)\left[\frac{2 m\left(n-m e^{-2 t}\right)}{\operatorname{sh} 2 t}-\frac{\left(n-m e^{-2 t}\right)^{2}}{s^{2} 2 t}\right] .
\end{aligned}
$$

Proof. Write

$$
y(t)=B_{n, m}(t)
$$

We have

$$
\begin{aligned}
\lambda y(t) & =\left\langle\phi_{n},\left(\Omega \phi_{m}\right) \circ a(t)\right\rangle \\
\Omega & =\frac{1}{4}\left(L_{V}^{2}+L_{Q}^{2}-L_{W}^{2}\right)
\end{aligned}
$$

Write

$$
N=\left(\begin{array}{ll}
0 & 0 \\
1 & 0
\end{array}\right) \in g, \quad U=\left(\begin{array}{ll}
0 & 1 \\
0 & 0
\end{array}\right) \in g
$$

We have

$$
\begin{gathered}
V=2 N+W, \quad[W, N]=Q \\
L_{V}^{2}=4 L_{N}^{2}+2 L_{N} L_{W}+2 L_{W} L_{N}+L_{W}^{2} \\
L_{W} L_{N}=L_{[W, N]}+L_{N} L_{W}=L_{Q}+L_{N} L_{W}
\end{gathered}
$$

Thus we have

$$
\begin{aligned}
& L_{V}^{2}=4 L_{N}^{2}+4 L_{N} L_{W}+2 L_{Q}+L_{W}^{2} \\
& 4 \Omega=4 L_{N}^{2}+4 L_{N} L_{W}+2 L_{Q}+L_{Q}^{2}
\end{aligned}
$$

This implies via (2.6)

$$
\left\langle\phi_{n},\left(L_{Q}^{2} \phi_{m}\right) \circ a(t)\right\rangle+2\left\langle\phi_{n},\left(L_{Q} \phi_{m}\right) \circ a(t)\right\rangle-4 \lambda y(t)=q_{1}(t)+q_{2}(t)
$$

where

$$
\begin{aligned}
& q_{1}(t)=4\left\langle\phi_{n},\left(L_{N}^{2} \phi_{m}\right) \circ a(t)\right\rangle \\
& q_{2}(t)=-4 i m\left\langle\phi_{n},\left(L_{N} \phi_{m}\right) \circ a(t)\right\rangle .
\end{aligned}
$$

Or

$$
y^{\prime \prime}+2 y^{\prime}-4 \lambda y=q_{1}(t)+q_{2}(t)
$$

by (2.3). We shall now find $q_{1}, q_{2}$. 
First let us show that

$$
L_{X}(v \circ g)=\left(L_{g}^{-1} X_{g} v\right) \circ g
$$

for every $g \in G, X \in g$ and every $C^{1}$-vector $v \in \mathbf{H}(T)$.

Indeed, we have

$$
\begin{aligned}
L_{X}(v \circ g) & =\lim _{t \rightarrow 0} \frac{T(\exp t X) T(g) v-T(g) v}{t} \\
& =\lim _{t \rightarrow 0} \frac{T(g) T\left(g^{-1} \cdot \exp t X \cdot g\right) v-T(g) v}{t} \\
& =T(g) \lim _{t \rightarrow 0} \frac{T\left(g^{-1} \cdot \exp t X \cdot g\right) v-v}{t}=\left(L_{g^{-1} \times g} v\right) \circ g .
\end{aligned}
$$

We have from (2.2) and (2.9)

$$
\begin{aligned}
\operatorname{iny}(t) & =\left\langle L_{W} \phi_{n}, \phi_{m} \circ a(t)\right\rangle=-\left\langle\phi_{n}, L_{W}\left(\phi_{m} \circ a(t)\right)\right\rangle \\
& =-\left\langle\phi_{n},\left(L_{a(t)^{-1} W a(t)} \phi_{m}\right) \circ a(t)\right\rangle .
\end{aligned}
$$

We have

$$
\begin{gathered}
W=U-N \\
a(t)^{-1} W a(t)=-e^{2 t} N+e^{-2 t} U=-2 \operatorname{sh} 2 t N+e^{-2 t} W
\end{gathered}
$$

and therefore

$$
\operatorname{iny}(t)=2 \operatorname{sh} 2 t\left\langle\phi_{n},\left(L_{N} \phi_{m}\right) \circ a(t)\right\rangle+i m e^{-2 t}\left\langle\phi_{n}, \phi_{m} \circ a(t)\right\rangle .
$$

Or

$$
q_{2}(t)=\frac{2 y(t) m\left(n-m e^{-2 t}\right)}{\operatorname{sh} 2 t} .
$$

Write

$$
z(t)=\left\langle\phi_{n},\left(L_{N} \phi_{m}\right) \circ a(t)\right\rangle
$$

and apply to $z(t)$ the above argument. We get

$$
-i n z(t)=-2 \operatorname{sh} 2 t\left\langle\phi_{n},\left(L_{N}^{2} \phi_{m}\right) \circ a(t)\right\rangle+e^{-2 t}\left\langle\phi_{n},\left(L_{W} L_{N} \phi_{m}\right) \circ a(t)\right\rangle .
$$

Here

$$
\left\langle\phi_{n},\left(L_{W} L_{N} \phi_{m}\right) \circ a(t)\right\rangle=y^{\prime}(t)-i m\left\langle\phi_{n},\left(L_{N} \phi_{m}\right) \circ a(t)\right\rangle=y^{\prime}(t)-i m z(t)
$$

by (2.7). This, (2.10) and (2.11) give

$$
q_{1}(t)=\frac{2 i z(t)\left(m e^{-2 t}-n\right)}{-\operatorname{sh} 2 t}+e^{-2 t} \frac{y^{\prime}(t)}{2 \operatorname{sh} 2 t}
$$

or

$$
q_{1}(t)=-\frac{y(t)\left(m e^{-2 t}-n\right)^{2}}{s h^{2} 2 t}+\frac{e^{-2 t} y^{\prime}(t)}{2 \operatorname{sh} 2 t} .
$$

This and (2.8) complete the proof.

It is well known [7] that

$$
B_{n, m}(t) \rightarrow 0 \quad \text { when } t \rightarrow \infty
$$

$n, m \in \mathbb{Z}$, since $T$ has no invariant vectors in $\mathbf{H}(T)$. 
We shall estimate for large $t$ the solution of (2.4) satisfying

$$
\begin{aligned}
y(t) & \rightarrow 0 \quad \text { when } t \rightarrow \infty \\
y(1) & =B_{n, m}(1) \\
y^{\prime}(1) & =B_{n, m}^{\prime}(1) .
\end{aligned}
$$

We have for $n, m \in \mathbb{Z}, t \in \mathbb{R}$

$$
\begin{aligned}
& \left|B_{n, m}(t)\right| \leq 1 \\
& \left|B_{n, m}^{\prime}(t)\right| \leq\left\|L_{Q} \phi_{m}\right\|
\end{aligned}
$$

by (2.3) and the Schwarz inequality. Also

$$
\left\langle\left(L_{V}^{2}+L_{Q}^{2}-L_{W}^{2}\right) \phi_{m}, \phi_{m}\right\rangle=4 \lambda
$$

if $\phi_{m} \neq 0$. This implies that for $\phi_{m} \neq 0$

$$
\left\|L_{\mathrm{v}} \phi_{m}\right\|^{2}+\left\|L_{\mathrm{Q}} \phi_{m}\right\|^{2}=m^{2}-4 \lambda
$$

and therefore

$$
\begin{gathered}
m^{2}-4 \lambda \geq 0 \\
\left|B_{n, m}^{\prime}(t)\right| \leq\left\|L_{Q} \phi_{m}\right\| \leq \sqrt{m^{2}-4 \lambda}
\end{gathered}
$$

for all $t \in \mathbb{R}, n, m \in \mathbb{Z}, \phi_{m} \neq 0$.

Let

$$
r_{1}=r_{1}(\lambda)=-1+\sqrt{1+4 \lambda}, \quad r_{2}=r_{2}(\lambda)=-1-\sqrt{1+4 \lambda},
$$

be the roots of the characteristic equation

$$
k^{2}+2 k-4 \lambda=0
$$

Here and henceforth $\sqrt{a}$ denotes the positive square root if $a>0$ and it denotes $i \sqrt{|a|}$ if $a<0$. Equation (2.4) can be written in the form

$$
\left(D-r_{1}\right)\left(D-r_{2}\right) y=f_{1}(t)+f_{2}(t)=f(t),
$$

where $D$ denotes the operator of differentiation and

$$
\begin{aligned}
& \left|f_{1}(t)\right| \leq C_{1} e^{-4 t} \sqrt{m^{2}-4 \lambda} \\
& \left|f_{2}(t)\right| \leq C_{2}\left(m^{2}+n^{2}\right) e^{-2 t}
\end{aligned}
$$

for some $C_{1}, C_{2}>0$ and all $t \geq 1$ by (2.5) and (2.13). We have

$$
\begin{gathered}
\left(D-r_{1}\right) y=e^{r_{1} t} D\left(e^{-r_{1} t} y\right) \\
e^{r_{1} t} D\left(e^{-r_{1} t}\left(D-r_{2}\right) y\right)=f(t) .
\end{gathered}
$$

This gives

$$
e^{\left(r_{2}-r_{1}\right) t} D\left(e^{-r_{2} t} y\right)=-\int_{t}^{\infty} e^{-r_{1} s} f(s) d s+P_{1}
$$

where $P_{1}$ is a constant. The integral on the right side of (2.15) converges by (2.14), since $\operatorname{Re}\left(r_{1}+2\right) \geq 1$. Also

$$
\begin{aligned}
& \left|\int_{t}^{\infty} e^{-r_{1} s} f_{1}(s) d s\right| \leq C_{1} \sqrt{m^{2}-4 \lambda} e^{-3 t} \\
& \left|\int_{1}^{\infty} e^{-r_{1} s} f_{2}(s) d s\right| \leq C_{2}\left(m^{2}+n^{2}\right) e^{-t}
\end{aligned}
$$


Expression (2.15) gives

$$
y=-e^{-t} \int_{1}^{t}\left(\int_{s}^{\infty} e^{u} f(u) d u\right) d s+P_{1} t e^{-t}+P_{2} e^{-t}
$$

if $r_{1}=r_{2}$ and

$$
\begin{aligned}
y & =e^{r_{2} t} A(t)+e^{r_{2} t}\left[P_{1} \int_{1}^{t} e^{\left(r_{1}-r_{2}\right) s} d s+P_{2}\right] \\
& =e^{r_{2} t} A(t)+\frac{P_{1}}{2 \sqrt{1+4 \lambda}} e^{r_{1} t}+\left(P_{2}-\frac{e^{2 \sqrt{1+4 \lambda}} P_{1}}{2 \sqrt{1+4 \lambda}}\right) e^{r_{2} t}
\end{aligned}
$$

if $r_{1} \neq r_{2}$, where

$$
\left|e^{r_{2} t} \int_{1}^{t} e^{\left(r_{1}-r_{2}\right) s} d s\right| \leq t e^{t \operatorname{Re} r_{1}}
$$

$P_{2}$ is a constant, and

$$
\begin{aligned}
A(t) & =-\int_{1}^{t} e^{\left(r_{1}-r_{2}\right) s}\left(\int_{s}^{\infty} e^{-r_{1} u} f_{1}(u) d u\right) d s-\int_{1}^{t} e^{\left(r_{1}-r_{2}\right) s}\left(\int_{s}^{\infty} e^{-r_{1} u} f_{2}(u) d u\right) d s \\
& =-\left(A_{1}(t)+A_{2}(t)\right) .
\end{aligned}
$$

We have using (2.14)

$$
\begin{aligned}
\left|e^{r_{2} t} A_{1}(t)\right| & \leq C_{1} \sqrt{m^{2}-4 \lambda} e^{t \operatorname{Re} r_{2}} \int_{1}^{t} e^{\left(\operatorname{Re} r_{1}-\operatorname{Re} r_{2}\right) s} e^{-\left(\operatorname{Re} r_{1}+2\right) s} d s \\
& \leq C_{1} \sqrt{m^{2}-4 \lambda} e^{t \operatorname{Re} r_{2}} e^{t\left(\operatorname{Re} r_{1}-\operatorname{Re} r_{2}\right)} \int_{1}^{t} e^{-\left(\operatorname{Re} r_{1}+2\right) s} d s \\
& \leq \bar{C}_{1} \sqrt{m^{2}-4 \lambda} e^{t \operatorname{Re} r_{1}}
\end{aligned}
$$

since $\operatorname{Re} r_{1}-\operatorname{Re} r_{2} \geq 0$ and $\operatorname{Re} r_{1}+2 \geq 1$.

Similarly we have

$$
\left|e^{r_{2} t} A_{2}(t)\right| \leq \bar{C}_{2}\left(m^{2}+n^{2}\right) e^{t \operatorname{Re} r_{1}}
$$

for some $\bar{C}_{2}>0$ and all $t \geq 1$. Also

$$
\begin{aligned}
& P_{1}=\int_{1}^{\infty} e^{-r_{1} s} f(s) d s-r_{2} e^{-r_{1}} y(1)+e^{-r_{1}} y^{\prime}(1) \\
& P_{2}=y(1) e^{-r_{2}}
\end{aligned}
$$

by (2.12), (2.15) and (2.17).

LEMMA 2.2. For $t \geq 1, n, m \in \mathbb{Z}$

$$
\left|B_{n, m}(t)\right| \leq K b(t)
$$

where

(1) $b(t)=\min \left\{t e^{-t}, e^{-t}\left(1+|1+4 \lambda|^{-1 / 2}\right)\right\}$, if $\lambda \leq-\frac{1}{4}$;

(2) $b(t)=\min \left\{t e^{r_{1} t}, e^{r_{1} t}|1+4 \lambda|^{-1 / 2}\right\}$ if $\lambda \in\left(-\frac{1}{4}, 0\right)$;

(3) $b(t)=t e^{-2 t}$ if $\lambda \geq 0$;

and $K=\bar{K}\left(m^{2}+n^{2}\right)+\tilde{K}, \bar{K}, \tilde{K}>0$ are independent of $\lambda$.

Proof. Cases (2) and (1) for $\lambda \in\left[-\frac{1}{2},-\frac{1}{4}\right]$ follow from expressions $(2.16)-(2.21)$, since

$$
\left|y^{\prime}(1)\right| \leq \sqrt{m^{2}-4 \lambda} \leq \sqrt{m^{2}+4} \text { and }|\sqrt{1+4 \lambda}| \leq 1 \text {. }
$$


Case 1 for $\lambda<-\frac{1}{2}$. In this case $\sqrt{m^{2}-4 \lambda}$ is unbounded and we cannot use (2.19) to estimate $e^{r_{2} t} A_{1}(t)$. Nevertheless we proceed as follows. We have

and

$$
A_{1}(t)=\int_{1}^{t} e^{\left(r_{1}-r_{2}\right) s}\left(\int_{s}^{\infty} e^{-r_{1} u} f_{1}(u) d u\right) d s
$$

$$
I(s)=\int_{s}^{\infty} e^{-r_{1} u} f_{1}(u) d u=2 \int_{s}^{\infty} e^{-\left(r_{1}+2\right) u} \frac{y^{\prime}(u)}{\operatorname{sh} 2 u} d u
$$

by $(2.5)$.

Let

$$
J(u)=\int_{u}^{\infty} \frac{y^{\prime}(v)}{\operatorname{sh} 2 v} d v
$$

Integration by parts gives

and therefore

$$
J(u)=\frac{y(u)}{\operatorname{sh} 2 u}+2 \int_{u}^{\infty} \frac{y(v) \operatorname{ch} 2 v}{\operatorname{sh}^{2} 2 v} d v
$$

$$
|J(u)| \leq Q_{1} e^{-2 u}
$$

for some $Q_{1}>0$ and all $u \geq 1$. Integrating by parts again we get

and

$$
I(s)=2\left[e^{-\left(r_{1}+2\right) s} J(s)+\left(r_{1}+2\right) \int_{s}^{\infty} e^{-\left(r_{1}+2\right) u} J(u) d u\right]
$$

$$
\begin{aligned}
A_{1}(t) & =2\left[\int_{1}^{t} e^{-\left(r_{2}+2\right) s} J(s) d s+\left(r_{1}+2\right) \int_{1}^{t} e^{\left(r_{1}-r_{2}\right) s}\left(\int_{s}^{\infty} e^{-\left(r_{1}+2\right) u} J(u) d u\right) d s\right] \\
& =2\left(F_{1}(t)+F_{2}(t)\right) .
\end{aligned}
$$

We have using (2.22)

$$
\left|e^{r_{2} t} F_{1}(t)\right|=\left|e^{r_{2} t} \int_{1}^{t} e^{-\left(r_{2}+2\right) s} J(s) d s\right| \leq Q_{1} e^{-t} \int_{1}^{t} e^{-3 s} d s \leq Q_{2} e^{-t}
$$

since $\operatorname{Re} r_{2}=-1$.

Now we shall estimate $e^{r_{2} t} F_{2}(t)$. We have

$$
e^{r_{2} t} F_{2}(t)=e^{r_{2} t}\left(r_{1}+2\right) \int_{1}^{t} e^{\left(r_{1}-r_{2}\right) s}\left(\int_{s}^{\infty} e^{-\left(r_{1}+2\right) u} J(u) d u\right) d s .
$$

Integration by parts gives

Here

$$
e^{r_{2} t} F_{2}(t)=\frac{e^{r_{2} t}\left(r_{1}+2\right)}{r_{1}-r_{2}}\left[\left.e^{\left(r_{1}-r_{2}\right) s} \int_{s}^{\infty} e^{-\left(r_{1}+2\right) u} J(u) d u\right|_{1} ^{t}+F_{1}(t)\right] .
$$

$$
\left|\frac{r_{1}+2}{r_{1}-r_{2}}\right|=\left|\frac{1+\sqrt{1+4 \lambda}}{2 \sqrt{1+4 \lambda}}\right| \leq 1
$$

since $\lambda<-\frac{1}{2}$. This and (2.24) give

$$
\left|e^{r_{2} t} F_{2}(t)\right| \leq Q_{3} e^{-t}
$$

since $\operatorname{Re} r_{2}=-1$. 
Expressions (2.24), (2.26) and (2.20) show that

$$
\left|e^{r_{2} t} A(t)\right| \leq\left(\bar{Q}_{1}\left(m^{2}+n^{2}\right)+\tilde{Q}\right) e^{-t}
$$

in (2.17) for some $\bar{Q}, \tilde{Q}>0$ and all $t \geq 1$. One can see from (2.21) that

$$
\left|\frac{P_{1}}{2 \sqrt{1+4 \lambda}}\right|,\left|P_{2}-\frac{e^{2 \sqrt{1+4 \lambda}} P_{1}}{2 \sqrt{1+4 \lambda}}\right|<\bar{Q}_{1}\left(m^{2}+n^{2}\right)+\tilde{Q}_{1}
$$

for some $\bar{Q}_{1}, \tilde{Q}_{1}>0$, since $\lambda<-\frac{1}{2}$. This completes case (1).

Case 3. In this case $\lambda \geq 0$ and $r_{1} \geq 0$. Therefore we cannot use (2.19) and (2.20). We proceed as follows. We have, using (2.14)

$$
\left|e^{r_{2} t} A_{2}(t)\right| \leq \frac{\bar{C}_{2}\left(m^{2}+n^{2}\right)}{r_{1}+2} e^{r_{2} t} \int_{1}^{t} e^{-\left(r_{2}+2\right) s} d s=\frac{\bar{C}_{2}\left(m^{2}+n^{2}\right)}{r_{1}+2} e^{r_{2} t} \cdot t \cdot e^{-\left(r_{2}+2\right) t}
$$

since $r_{2} \leq-2$.

Similarly

$$
\left|e^{r_{2} t} A_{1}(t)\right| \leq \frac{C_{4} \sqrt{m^{2}-4 \lambda}}{r_{1}+2} e^{r_{2} t} \int_{1}^{t} e^{-\left(r_{2}+2\right) s} d s \leq \bar{C}_{4} m \cdot t e^{-2 t}
$$

by (2.14), since $\lambda \geq 0$. Here $m^{2}-4 \lambda \geq 0$ if $\phi_{m} \neq 0$ by (2.13). This and (2.27) imply that

$$
\left|e^{r_{2} t} A(t)\right| \leq\left(\bar{C}\left(m^{2}+n^{2}\right)+\tilde{C}\right) t e^{-2 t}
$$

in (2.17) for some $\bar{C}, \tilde{C}>0$ and all $t \geq 1$.

Since $y(t) \rightarrow 0$ when $t \rightarrow \infty$ and $r_{1} \geq 0$ we have

$$
P_{1}=0
$$

in (2.17). Thus

$$
y(t)=e^{r_{2} t} A(t)+y(1) e^{-r_{2}} e^{r_{2} t}
$$

by (2.17) and (2.21). Therefore

$$
|y(t)| \leq\left(\bar{C}_{1}\left(m^{2}+n^{2}\right)+\tilde{C}_{1}\right) t e^{-2 t}
$$

for some $\bar{C}_{1}, \tilde{C}_{1}>0$ and all $t \geq 1$ by (2.28). This completes the proof of the lemma.

LEMMA 2.3. Let $C_{n, m}(t)=\left\langle\phi_{n}, \phi_{m} \circ u(t)\right\rangle$ where $u(t)$ is defined in (2). Then for all $t \geq 1$

$$
\left|C_{n, m}(t)\right| \leq K b(\ln t)
$$

where $b(t)$ and $K$ are as in lemma $2.2, n, m \in \mathbb{Z}$.

Proof. One can show that

$$
u(t)=r\left(\theta_{2}\right) a(\ln t) r\left(\theta_{1}\right)
$$

for some $\theta_{1}=\theta_{1}(t), \theta_{2}=\theta_{2}(t) \in(-\pi, \pi]$. To do so, one can use either the Cartan decomposition of $G$ or simply the geometrical model of $G$ as the unit tangent bundle of the hyperbolic plane. In fact, Hedlund showed this relation geometrically in his proof of ergodicity of the horocycle flow (see [6]).

We have

$$
\begin{aligned}
C_{n, m}(t) & =\left\langle\phi_{n}, \phi_{m} \circ u(t)\right\rangle=\left\langle\phi_{n}, \phi_{m} \circ r\left(\theta_{1}\right) \circ a(\ln t) \circ r\left(\theta_{2}\right)\right\rangle \\
& =\left\langle\phi_{n} \circ r\left(-\theta_{2}\right), \phi_{m} \circ r\left(\theta_{1}\right) \circ a(\ln t)\right\rangle=e^{-i n \theta_{2}} e^{i m \theta_{1}}\left\langle\phi_{n}, \phi_{m} \circ a(\ln t)\right\rangle .
\end{aligned}
$$


Thus

$$
\left|C_{n, m}(t)\right|=\left|B_{n, m}(\ln t)\right| \leq K b(\ln t)
$$

by lemma 2.2 .

Proof of theorem 1. Let $T$ be a non-trivial irreducible unitary representation of $G$ in $\mathbf{H}(T)$ and let $\left\{\phi_{n}, n \in \mathbb{Z}\right\}$ be as above. Let $v, w \in K(T, p), p>0$. We have

$$
v=\sum_{n \in \mathbf{Z}} c_{n} \phi_{n}, \quad w=\sum_{n \in \mathbf{Z}} d_{n} \phi_{n} .
$$

where $c_{n}=\left\langle v, \phi_{n}\right\rangle, d_{n}=\left\langle w, \phi_{n}\right\rangle, n \in \mathbb{Z}$.

Consider the following possibilities for $p$.

(1) Let $p \geq 3$. We have

$$
B(t)=\langle v, w \circ a(t)\rangle=\sum_{n, m \in Z} c_{n} d_{m}\left\langle\phi_{n}, \phi_{m} \circ a(t)\right\rangle=\sum c_{n} d_{m} B_{n, m}(t) .
$$

Therefore

$$
|B(t)| \leq b(t) \sum_{n, m}\left|c_{n}\right|\left|d_{m}\right|\left(\bar{K}\left(m^{2}+n^{2}\right)+\tilde{K}\right)=b(t) \cdot S
$$

by lemma 2.2 .

Standard elementary argument shows that $S$ is finite. Indeed, we have

$$
S \leq K^{\prime}\left(\sum_{m, n \in \mathbf{Z}}\left|c_{n}\right|\left|d_{m}\right| m^{2}+\sum_{m, n \in \mathbf{Z}}\left|c_{n}\right|\left|d_{m}\right| n^{2}\right)
$$

for some $K^{\prime}>0$.

We have, using corollary 1.1 and the Schwarz inequality,

$$
\begin{aligned}
& \sum_{m \in \mathbf{Z}}\left|d_{m}\right| m^{2}=\sum_{0 \neq m \in Z} \frac{|m|^{3}\left|d_{m}\right|}{|m|} \leq\left(\sum_{m \in \mathbf{Z}} m^{6}\left|d_{m}\right|^{2}\right)^{1 / 2} \cdot\left(\sum_{0 \neq m \in \mathbf{Z}} \frac{1}{m^{2}}\right)^{1 / 2} \\
&=C_{1}\left\|L_{W}^{3} \omega\right\|<\infty, \\
& \sum_{n \in \mathbf{Z}}\left|c_{n}\right| \leq\left(\sum_{n \in Z} n^{6}\left|c_{n}\right|^{2}\right)^{1 / 2} \cdot\left(\sum_{0 \neq n \in Z} \frac{1}{n^{6}}\right)^{1 / 2}+\left|c_{0}\right|=C_{2}\left\|L_{W}^{3} v\right\|+\left|c_{0}\right|,
\end{aligned}
$$

since $p \geq 3$. Thus $\alpha(p)=1$ for $p \geq 3$.

(2) Let $2 \leq p<3$. Let $0<\delta<1$ be chosen later. Write

$$
\bar{v}_{t}=\sum_{|n| \leq(b(t))^{-\delta}} c_{n} \phi_{n}, \quad \hat{v}_{t}=\sum_{|n|>(b(t))^{-\delta}} c_{n} \phi_{n} .
$$

We have using corollary 1.2

$$
\left\|\hat{v}_{t}\right\|=\left(\sum_{|n|>(b(t))^{-\delta}}\left|c_{n}\right|^{2}\right)^{1 / 2} \leq D(b(t))^{\delta p} .
$$

We have

$$
B(t)=\langle v, w \circ a(t)\rangle=\left\langle\bar{v}_{t}, w \circ a(t)\right\rangle+\left\langle\hat{v}_{t}, w \circ a(t)\right\rangle=\bar{V}+\hat{V}
$$

Also

$$
\bar{V}=\left\langle\bar{v}_{t}, \bar{w}_{t} \circ a(t)\right\rangle+\left\langle\bar{v}_{t}, \hat{w}_{t} \circ a(t)\right\rangle=\bar{W}+\hat{W}
$$

and

$$
\begin{aligned}
|\hat{V}| & \leq\left\|\hat{v}_{t}\right\| \cdot\|w\| \leq D_{1}\|w\|(b(t))^{\delta p} \\
|\hat{W}| & \leq\|v\| \cdot\left\|\hat{w}_{t}\right\| \leq D_{2}\|v\|(b(t))^{\delta p}
\end{aligned}
$$


284

M. Rather

by (2.29). For $\bar{W}$ we have

$$
\begin{aligned}
|\bar{W}| & \leq \sum_{|m|,|n| \leq(b(t))^{-\delta}}\left|c_{n}\right|\left|d_{m}\right| B_{n, m}(t) \\
& \leq b(t) K^{\prime}\left(\sum_{|m|,|n| \leq(b(t))^{-\delta}}\left|c_{n}\right|\left|d_{m}\right| \cdot m^{2}+\sum_{|m|,|n| \leq(b(t))^{-\delta}}\left|c_{n}\right|\left|d_{m}\right| \cdot n^{2}\right)
\end{aligned}
$$

for some $K^{\prime}>0$ by lemma 2.2. Since $p \geq 2$ we have

$$
\begin{aligned}
& \sum_{|m| \leq(b(t))^{-\delta}}\left|d_{m}\right| m^{2} \leq(b(t))^{-\delta / 2}\left(\sum_{m \in \mathbb{Z}}\left|d_{m}\right|^{2} m^{4}\right)^{1 / 2}=(b(t))^{-\delta / 2}\left\|L_{W}^{2} w\right\| \\
& \sum_{|n| \leq\{b(t))^{-\delta}}\left|c_{n}\right| \leq\left(\sum_{0 \neq n \in \mathbb{Z}} \frac{1}{n^{4}}\right)^{1 / 2}\left(\sum_{n \in \mathbb{Z}} n^{4}\left|c_{n}\right|^{2}\right)^{1 / 2}+\left|c_{0}\right|=Q_{1}\left\|L_{W}^{2} v\right\|+\left|c_{0}\right| .
\end{aligned}
$$

Thus

$$
|\bar{W}| \leq Q_{2}(b(t))^{1-(\delta / 2)}
$$

by $(2.31)$ and

$$
|B(t)| \leq Q_{3}\left[(b(t))^{\delta p}+(b(t))^{1-(\delta / 2)}\right]
$$

by (2.30). Here $Q_{2}, Q_{3}>0$ depend only on $v, w$.

Now choose $\delta=2 /(2 p+1)$ to get

$$
|B(t)| \leq 2 Q_{3}(b(t))^{2 p /(2 p+1)} .
$$

Thus $\alpha(p)=2 p /(2 p+1)$ for $2 \leq p<3$.

(3) Let $1 \leq p<2$. We proceed as in (2) changing estimates in (2.32). Since $p \geq 1$ we have

$$
\begin{gathered}
\sum_{|m| \leq(b(t))^{-\delta}}\left|d_{m}\right| m^{2} \leq\left(\sum_{|m| \leq(b(t))^{-\delta}} m^{2}\right)^{1 / 2} \cdot\left(\sum_{m \in \mathbf{Z}}\left|d_{m}\right|^{2} m^{2}\right)^{1 / 2} \leq L_{1}(b(t))^{-3 \delta / 2}\left\|L_{W} w\right\| \\
\sum_{|n| \leq(b(t))^{-\delta}}\left|c_{n}\right| \leq\left(\sum_{0 \neq n \in \mathbb{N}} \frac{1}{n^{2}}\right)^{1 / 2} \cdot\left(\sum_{n \in \mathbf{Z}} n^{2}\left|c_{n}\right|^{2}\right)^{1 / 2}+\left|c_{0}\right|=L_{2}\left\|L_{W} v\right\|+\left|c_{0}\right| .
\end{gathered}
$$

Thus

$$
|\bar{W}| \leq L_{3}(b(t))^{1-\frac{3}{2} \delta}
$$

and

$$
|B(t)| \leq L_{4}\left[b(t)^{\delta p}+(b(t))^{1-\frac{3}{2} \delta}\right] .
$$

Setting $\delta=2 /(2 p+3)$ we get that $\alpha(p)=2 p /(2 p+3)$ if $1 \leq p<2$.

(4) Let $0<p<1$. Using lemma 1.3 for $p$ we get

$$
\left\|\hat{v}_{t}\right\| \leq(b(t))^{\delta p}
$$

and therefore

$$
|\hat{V}|,|\hat{W}| \leq D(b(t))^{\delta p}
$$

For $\bar{W}$ we get

$$
\begin{gathered}
\sum_{|m| \leq(b(t))^{-\delta}}\left|d_{m}\right| m^{2} \leq\left(\sum_{|m| \leq(b(t))^{-\delta}} m^{4}\right)^{1 / 2} \cdot\left(\sum_{m \in \mathbf{Z}}\left|d_{m}\right|^{2}\right)^{1 / 2} \leq P_{1}(b(t))^{-5(\delta / 2)}\|w\| \\
\sum_{|n| \leq(b(t))^{-\delta}}\left|c_{n}\right| \leq(b(t))^{-\delta / 2} \cdot\left(\sum_{n \in \mathbf{Z}}\left|c_{n}\right|^{2}\right)^{1 / 2}=(b(t))^{-\delta / 2}\|v\| .
\end{gathered}
$$

https://doi.org/10.1017/S0143385700004004 Published online by Cambridge University Press 
Thus

$$
|\bar{W}| \leq P_{2}(b(t))^{1-3 \delta}
$$

and

$$
|B(t)| \leq P_{3}\left[(b(t))^{\delta p}+b(t)^{1-3 \delta}\right] .
$$

Setting $\delta=1 /(p+3)$ we get that $\alpha(p)=p /(p+3)$.

This completes the proof of theorem 1 for $B(t)$. The proof for $C(t)$ is the same via lemma 2.3 .

Proof of theorem 3. Let $T$ be a unitary representation of $G$ having no non-zero invariant vectors in $\mathbf{H}(T)$. By fact $1.1, T$ is unitary equivalent to a direct integral $\pi$ of non-trivial unitary irreducible representations $\pi_{y}, y \in Y$ defined in $\S 1$. It is clear that

$$
\Lambda\left(\Omega_{T}\right)=\Lambda\left(\Omega_{\pi}\right)
$$

For $y \in Y$ let $\Omega(y)$ be the Casimir operator associated with $\pi_{y}$. Then

$$
\Omega(y) v=\lambda(y) v
$$

for some $\lambda(y) \in \mathbb{R}$ and every $C^{2}$-vector $v \in \mathbf{H}(y), y \in Y$. The function $\lambda$ is a measurable real-valued function on $Y$ and the spectrum $\Lambda\left(\Omega_{\pi}\right)$ is exactly the essential range of $\lambda$, i.e.

$$
\Lambda=\Lambda\left(\Omega_{\pi}\right)=\left\{x \in \mathbb{R}: \nu\left(\lambda^{-1}(x-\delta, x+\delta)\right)>0 \text { for all } \delta>0\right\} .
$$

We can assume without loss of generality that $Y=\lambda^{-1}(\Lambda)$.

It is clear that a number $x \in \Lambda$ is an eigenvalue of $\Omega_{\pi}$ if and only if $\nu\left(\lambda^{-1}\{x\}\right)>0$. Let

$$
A(T)=A(\pi)=\Lambda \cap\left(-\frac{1}{4}, 0\right)
$$

For $y \in Y$ and $t \geq 1$ let

$$
b_{y}(t)=b_{\lambda(y)}(t)
$$

where $b_{\lambda(y)}(t)$ is as in theorem 1 .

(1) Let $A(\pi) \neq \varnothing,-\frac{1}{4}<\beta(\pi)<0$ and $\sigma=\sigma(\pi)=-1+\sqrt{1+4 \beta(\pi)}>-1$ be as in theorem 3. Let

$$
\begin{aligned}
& \bar{\beta}=-\left(\beta(\pi)+\frac{1}{4}\right) / 2 \\
& \bar{\sigma}=-1+\sqrt{1+4 \bar{\beta}}<\sigma \\
& C=1 / \sqrt{1+4 \bar{\beta}} .
\end{aligned}
$$

Also let

$$
t_{0}=\inf \left\{t \geq 1: t e^{\bar{\sigma} t}<e^{\sigma t}\right\}
$$

We have using theorem 1

$$
b_{y}(t) \leq C e^{\sigma t}
$$

for all $t \geq t_{0}$ and a.e. $y \in Y$. We set in this case

$$
b_{\pi}(t)=C e^{\sigma t}
$$

(2) $A(\pi)=\varnothing, \tau=\sup \left(\Lambda \cap\left(-\infty,-\frac{1}{4}\right)\right)<-\frac{1}{4}$ and $\nu\left(\lambda^{-1}\left\{-\frac{1}{4}\right\}\right)=0$. Then

$$
C_{\tau}=\left(1+|1+4 \tau|^{-1 / 2}\right)<\infty
$$


and theorem 1 shows that

$$
b_{y}(t) \leq C_{\tau} e^{-t}
$$

for all $t \geq 1$ and a.e. $y \in Y$. We set in this case

$$
b_{\pi}(t)=C_{\tau} e^{-t} \text {. }
$$

(3) $A(\pi)=\varnothing$ and either $\tau=-\frac{1}{4}$ or $\nu\left(\lambda^{-1}\left\{-\frac{1}{4}\right\}\right)>0$. By theorem 1

$$
b_{y}(t) \leq t e^{-t}
$$

for all $t \geq 1$ and a.e. $y \in Y$. We set

$$
b_{\pi}(t)=t e^{-t}
$$

(4) $\Lambda \subset[0, \infty)$. Then

$$
b_{y}(t) \leq t e^{-2 t}=b_{\pi}(t)
$$

for all $t \geq 1$ and a.e. $y \in Y$.

From now we proceed just as in the proof of theorem 1.

Let $\left\{\phi_{n}(y), n \in \mathbb{Z}\right\}-\{0\}$ be the orthonormal basis in $\mathbf{H}(y), y \in Y$ found in lemma 1.1. It satisfies (1.1).

Let $v, w \in K(\pi, p), p>0$. Consider the following possibilities for $p$

(i) Let $p \geq 3$. We have

$$
\begin{aligned}
v(y) & =\sum_{n \in \mathbf{Z}} c_{n}(y) \phi_{n}(y) \\
w(y) & =\sum_{n \in \mathbf{Z}} d_{n}(y) \phi_{n}(y),
\end{aligned}
$$

where $c_{n}(y)=\left\langle v(y), \phi_{n}(y)\right\rangle, d_{n}(y)=\left\langle w(y), \phi_{n}(y)\right\rangle, y \in Y$. Write

$$
B_{n, m}^{y}(t)=\left\langle\phi_{n}(y), \phi_{m}(y) \circ a(t)\right\rangle \text {. }
$$

We have

$$
\begin{aligned}
B(t) & =\langle v, w \circ a(t)\rangle=\int_{Y}\langle v(y), w(y) \circ a(t)\rangle d \nu(y) \\
& =\int_{Y}\left(\sum_{n, m \in \mathbb{Z}} c_{n}(y) d_{m}(y) B_{n, m}^{y}(t)\right) d \nu(y) .
\end{aligned}
$$

By lemma 2.2 for $t \geq t_{0}$

$$
\left|B_{n, m}^{y}(t)\right| \leq K b_{y}(t) \leq K b_{\pi}(t)
$$

for a.e. $y \in Y$ where $K=K\left(m^{2}+n^{2}\right)+\tilde{K}$ and $\bar{K}, \tilde{K}>0$ are independent of $y$. Thus

$$
|B(t)| \leq b_{\pi}(t) \int_{Y}\left(\sum_{m, n \in \mathbf{Z}}\left|c_{n}(y)\right|\left|d_{m}(y)\right|\left(\bar{K}\left(m^{2}+n^{2}\right)+\tilde{K}\right)\right) d \nu(y)=b_{\pi}(t) \cdot S
$$

where

$$
\begin{aligned}
S & \leq K^{\prime}\left[\int_{Y}\left(\sum_{n, m \in \mathbf{Z}}\left|c_{n}(y) \| d_{m}(y)\right| m^{2}\right) d \nu(y)+\int_{Y}\left(\sum_{n, m \in \mathbf{Z}}\left|c_{n}(y)\right|\left|d_{m}(y)\right| n^{2}\right) d \nu(y)\right] \\
& =K^{\prime}\left(S_{1}+S_{2}\right) .
\end{aligned}
$$

By lemma 1.2

$$
v(y), w(y) \in K\left(\pi_{y}, 3\right)
$$


for a.e. $y \in Y$. As in the proof of theorem 1 we have

$$
\begin{aligned}
\sum_{m \in \mathbf{Z}}\left|d_{m}(y)\right| m^{2} & \leq C_{1}\left\|L_{W}^{3} w(y)\right\| \\
\sum_{n \in Z}\left|c_{n}(y)\right| & \leq C_{2}\left\|L_{W}^{3} v(y)\right\|+\left|c_{0}(y)\right|
\end{aligned}
$$

for some $C_{1}, C_{2}>0$. Thus

$$
\begin{aligned}
\left|S_{1}\right| & \leq C_{1} \cdot C_{2} \cdot \int_{Y}\left\|L_{W}^{3} v(y)\right\| \cdot\left\|L_{W}^{3} w(y)\right\| d \nu(y)+C_{1} \int_{Y}\left\|L_{W}^{3} w(y)\right\| \| c_{0}(y) \mid d \nu(y) \\
& \leq C_{3}\left\|L_{W}^{3} v\right\| \cdot\left\|L_{W}^{3} w\right\|+C_{1}\left\|L_{W}^{3} w\right\| \cdot\|v\|
\end{aligned}
$$

by the Schwarz inequality. This proves that

$$
B(t) \leq C_{4} b_{\pi}(t)
$$

for all $t \geq t_{0}$ and some $C_{4}>0$. Thus $\alpha(p)=1$ for $p \geq 3$.

(ii) Let $2 \leq p<3$. Let $\delta>0$ be chosen later and let $t \geq t_{0}$.

Let $\bar{v}, \hat{v} \in \mathbf{H}(\pi)$ be defined by

$$
\begin{aligned}
& \bar{v}_{t}(y)=\sum_{|n| \leq\left\{b_{\pi}(t)\right)^{-\delta}} c_{n}(y) \phi_{n}(y), \\
& \hat{v}_{t}(y)=\sum_{|n|>\left(b_{\pi}(t)\right)^{-\delta}} c_{n}(y) \phi_{n}(y),
\end{aligned}
$$

$y \in Y$. We have

$$
\begin{gathered}
v=\bar{v}_{t}+\hat{v}_{t} \\
\left\|\hat{v}_{t}\right\|=\left(\int_{Y}\left(\sum_{|n|>\left(b_{\pi}(t)\right)^{-\delta}}\left\|c_{n}(y)\right\|^{2}\right) d \nu(y)\right)^{1 / 2} \leq D\left(b_{\pi}(t)\right)^{\delta p}
\end{gathered}
$$

by corollary 1.2 .

We have

$$
B(t)=\langle v, w \circ a(t)\rangle=\left\langle\bar{v}_{t}, w \circ a(t)\right\rangle+\left\langle\hat{v}_{t}, w \circ a(t)\right\rangle=\bar{V}+\hat{V} .
$$

Also

$$
\bar{V}=\left\langle\bar{v}_{t}, \bar{w}_{t} \circ a(t)\right\rangle+\left\langle\bar{v}_{t}, \hat{w}_{\imath} \circ a(t)\right\rangle=\bar{W}+\hat{W}
$$

and

$$
\begin{aligned}
|\hat{V}| & \leq\left\|\hat{v}_{t}\right\| \cdot\|w\| \leq D_{1}\|w\|\left(b_{\pi}(t)\right)^{\delta p} \\
|\hat{W}| & \leq\|v\| \cdot\left\|\hat{w}_{t}\right\| \leq D_{2}\|v\|\left(b_{\pi}(t)\right)^{\delta p} .
\end{aligned}
$$

For $\bar{W}$ we have

$$
\begin{aligned}
|\bar{W}| & \leq \int_{Y}\left(\sum_{|m|,|n| \leq\left(b_{\pi}(t)\right)^{-\delta}}\left|c_{n}(y)\right|\left|d_{m}(y)\right|\left|B_{n, m}^{y}(t)\right|\right) d \nu(y) \\
& \leq b_{\pi}(t) \cdot K^{\prime} \int_{Y}\left(\sum_{|m|,|n| \leq\left(b_{m}(t)\right)^{\delta}}\left|c_{n}(y)\right|\left|d_{m}(y)\right|\left(m^{2}+n^{2}\right)\right) d \nu(y)
\end{aligned}
$$

for some $K^{\prime}>0$ by lemma 2.2 .

It follows from lemma 1.2 that

$$
v(y), w(y) \in K\left(\pi_{y}, 2\right)
$$


for a.e. $y \in Y$. For these $y$ we have

$$
\begin{aligned}
& \sum_{|m| \leq\left(b_{\pi}(t)\right)^{-\delta}}\left|d_{m}(y)\right| m^{2} \leq\left(b_{\pi}(t)\right)^{-\delta / 2}\left(\sum_{m \in \mathbf{Z}} m^{4}\left|d_{m}(y)\right|^{2}\right)^{1 / 2}=\left(b_{n}(t)\right)^{-\delta / 2}\left\|L_{W}^{2} w(y)\right\| \\
& \sum_{|n| \leq\left(b_{n}(t)\right)^{-\delta}}\left|c_{n}(y)\right| \leq\left(\sum_{0 \neq n \in \mathbf{Z}} \frac{1}{n^{4}}\right)^{1 / 2} \cdot\left(\sum_{n \in \mathbf{Z}} n^{4}\left|c_{n}(y)\right|^{2}\right)^{1 / 2}+\left|c_{0}(y)\right| \\
&=Q_{1}\left\|L_{W}^{2} v(y)\right\|+\left|c_{0}(y)\right|
\end{aligned}
$$

since $p \geq 2$. Thus

$$
\begin{aligned}
|\bar{W}| & \leq Q_{2}\left(b_{\pi}(t)\right)^{1-(\delta / 2)} \int_{Y}\left\|L_{W}^{2} w(y)\right\|\left(\left\|L_{W}^{2} v(y)\right\|+\left|c_{0}(y)\right|\right) d \nu(y) \\
& \leq Q_{2}\left(b_{\pi}(t)\right)^{1-(\delta / 2)}\left\|L_{W}^{2} w\right\|\left(\left\|L_{W}^{2} v\right\|+\|v\|\right) .
\end{aligned}
$$

This and (2.33) imply

$$
|B(t)| \leq Q_{3}\left[\left(b_{\pi}(t)\right)^{\delta p}+\left(b_{\pi}(t)\right)^{1-(\delta / 2)}\right] .
$$

We choose $\delta=2 /(2 p+1)$ to get

$$
|B(t)| \leq 2 Q_{3}\left(b_{\pi}(t)\right)^{2 p /(2 p+1)} .
$$

Thus $\alpha(p)=2 p /(2 p+1)$ for $2 \leq p<3$.

We have just shown how to adjust the proof of theorem 1 to the case of the direct integral. It is now clear that cases $1 \leq p<2$ and $0<p<1$ can be handled similarly. This and lemma 2.3 complete the proof of the theorem.

\section{REFERENCES}

[1] A. Borel \& N. Wallach. Continuous Cohomology, Discrete Subgroups, and Representations of Reductive Groups. Annals of Math. Studies 94 (1980).

[2] P. Collet, H. Epstein \& G. Gallavotti. Perturbations of geodesic flows on surfaces of constant negative curvature and their mixing properties. Commun. Math. Phys. 95 61-112 (1984).

[3] S. Gelbart. Automorphic Forms on Adele Groups. Annals of Math. Studies 83 (1975).

[4] I. Gelfand, M. Graev \& I. Pyatetskii-Shapiro. Representation Theory and Automorphic Functions. W. B. Saunders Co., Philadelphia, 1969.

[5] V. Guillemin \& D. Kazhdan. Some inverse spectral results for negatively curved 2-manifolds. Topology 19 301-312 (1980).

[6] E. Hopf. Statistik der geodätischen Linien in Manigfaltigkeiten negativer Krümung. Ber. Voch. Sachs. Akad. Wiss. Leipzig 91 (1939), 261-304.

[7] R. Howe \& C. Moore. Asymptotic properties of unitary representations. J. Funct. Anal. 32 (1979), $72-96$.

[8] Y. Katznelson. An Introduction to Harmonic Analysis. Dover Publications, 1976.

[9] T. Kubota. Elementary Theory of Eisenstein Series. Halsted Press, 1973.

[10] S. Lang. Real Analysis. Addison-Wesley, 1969.

[11] S. Lang. $\mathrm{SL}_{2}(\mathbb{R})$. Springer-Verlag, 1985.

[12] P. Lax \& R. Phillips. The asymptotic distribution of lattice points in Euclidean and non Euclidean spaces. J. Funct. Anal. 46 (1983), \#3.

[13] G. Mackey. The Theory of Unitary Group Representations. Univ, of Chicago Press, 1976.

[14] D. Milicic. Asymptotic behavior of matrix coefficients of the discrete series. Duke Math. J.44 (1977), 59-88.

[15] S. Patterson. The limit set of a Fuchsian group. Acta Math. 136 (1976), 241-273.

[16] P. Trombi. Asymptotic expansions of matrix coefficients: The real rank one case. J. Funct. Anal. 30 (1978), 83-105. 